\title{
Real-time observation of disintegration processes within argon clusters ionized by a hard-x-ray pulse of moderate fluence
}

\author{
Yoshiaki Kumagai $\odot,{ }^{1,2, *}$ Zoltan Jurek, ${ }^{3,4}$ Weiqing Xu $\odot,{ }^{1,5}$ Hironobu Fukuzawa $\odot,{ }^{1,6}$ Koji Motomura, ${ }^{1}$ Denys Iablonskyi $\odot,{ }^{1}$ \\ Kiyonobu Nagaya, ${ }^{6,7}$ Shin-ichi Wada $\odot,{ }^{6,8}$ Subhendu Mondal $\odot,{ }^{1}$ Tetsuya Tachibana, ${ }^{1}$ Yuta Ito, ${ }^{1}$ Tsukasa Sakai, ${ }^{7}$ \\ Kenji Matsunami, ${ }^{7}$ Toshiyuki Nishiyama ${ }^{7}{ }^{7}$ Takayuki Umemoto, ${ }^{8}$ Christophe Nicolas, ${ }^{9}$ Catalin Miron $\odot,{ }^{10,9,11}$ \\ Tadashi Togashi, ${ }^{12}$ Kanade Ogawa $\odot,{ }^{6}$ Shigeki Owada, ${ }^{6}$ Kensuke Tono ${ }^{\circ},{ }^{12}$ Makina Yabashi $\odot,{ }^{6}$ Sang-Kil Son $\odot,{ }^{3,4}$ \\ Beata Ziaja ${ }^{3},{ }^{3,13}$ Robin Santra $\odot, 3,4,14$ and Kiyoshi Ueda ${ }^{1,6}$ \\ ${ }^{1}$ Institute of Multidisciplinary Research for Advanced Materials, Tohoku University, Sendai 980-8577, Japan \\ ${ }^{2}$ Department of Applied Physics, Tokyo University of Agriculture and Technology, Koganei-shi, Tokyo 184-8588, Japan \\ ${ }^{3}$ Center for Free-Electron Laser Science, Deutsches Elektronen-Synchrotron, Notkestrasse 85, 22607 Hamburg, Germany \\ ${ }^{4}$ The Hamburg Centre for Ultrafast Imaging, Luruper Chaussee 149, 22671 Hamburg, Germany \\ ${ }^{5}$ School of Physical Science and Technology, ShanghaiTech University, Shanghai 201210, China \\ ${ }^{6}$ RIKEN SPring-8 Center, Sayo, Hyogo 679-5148, Japan \\ ${ }^{7}$ Department of Physics, Kyoto University, Kyoto 606-8502, Japan \\ ${ }^{8}$ Department of Physical Science, Hiroshima University, Higashi-Hiroshima 739-8526, Japan \\ ${ }^{9}$ Synchrotron SOLEIL, L'Orme des Merisiers, Saint-Aubin, BP 48, FR-91192 Gif-sur-Yvette Cedex, France \\ ${ }^{10}$ Université Paris-Saclay, CEA, CNRS, LIDYL, 91191, Gif-sur-Yvette, France \\ ${ }^{11}$ Extreme Light Infrastructure-Nuclear Physics, "Horia Hulubei” National Institute for Physics and Nuclear Engineering, \\ 30 Reactorului Street, RO-077125 Mâgurele, Judetul Ilfov, Romania \\ ${ }^{12}$ Japan Synchrotron Radiation Research Institute, Sayo, Hyogo 679-5198, Japan \\ ${ }^{13}$ Institute of Nuclear Physics, PAS, Radzikowskiego 152, 31-342, Krakow, Poland \\ ${ }^{14}$ Department of Physics, Universität Hamburg, Jungiusstrasse 9, 20355 Hamburg, Germany
}

(Received 9 September 2019; accepted 16 January 2020; published 25 February 2020)

\begin{abstract}
We present a time-resolved study of disintegration within atomic clusters ionized by a hard-x-ray pulse of moderate fluence. It was performed with electron and ion spectroscopy, and complemented by theoretical simulations. The expanding clusters were probed with a near-infrared (NIR) laser pulse over a range of pump-probe delays from -4 to $10 \mathrm{ps}$. In addition to an increasing number of singly charged atomic ions, originating from the ionization of Rydberg atoms formed through electron-ion recombination, we observe a decrease of oligomer yields. The latter is due to the interaction of oligomers with the NIR probe pulse, leading to their dissociation. At time delays between -1 and 2 ps, efficient absorption of the NIR laser energy occurs, even though the NIR intensity is too low to trigger tuneling ionization of Ar atoms. Our observations are similar to earlier observations of the fragmentation behavior of clusters excited by soft-x-ray pulses. This indicates that the relaxation dynamics of $\mathrm{x}$-ray-excited nano-objects are universal over a wide range of excitation photon energies.
\end{abstract}

DOI: 10.1103/PhysRevA.101.023412

\section{INTRODUCTION}

X-ray free-electron lasers (XFELs) [1,2] provide opportunities to investigate the structure of matter at near-atomic resolution [3-5], to track ultrafast transient processes in matter on femtosecond timescales [6-8], and to explore nonlinear phenomena induced by high-intensity X-ray pulses [9-11]. The operation of XFEL facilities in recent years [12-14] has been driving the science and technology of XFELs to new frontiers. Therefore, understanding the details of the interaction between an intense x-ray pulse and matter, in particular, in a time-resolved way, is of great importance for numerous applications using XFELs [15,16].

\footnotetext{
*kumagai@go.tuat.ac.jp
}

Studies of atomic gases irradiated with intense $\mathrm{x}$-ray pulses have shown that irradiated single atoms can reach a very high degree of ionization through sequential multiphoton absorption $[9,17,18]$. In case of nanosized bonded atomic assemblies irradiated by $\mathrm{x}$ rays, hot nanoplasmas are formed [19-21]. They quickly disintegrate through the Coulomb explosion of the highly charged constituent ions.

Interestingly, the ionization dynamics of such irradiated nano-objects significantly change at moderate pulse fluence. Instead of the rapid fragmentation into single atomic ions, chemical processes start to dominate the dynamics, due to the interatomic bonding "surviving" to a large extent the x-ray exposure. They distribute the increasing net charge within the sample and induce bond reorganization which leads to the formation of oligomers [22]. Achieving control over the disintegration dynamics of nanosized objects (triggered by 
X-ray pulses of moderate fluence) may potentially provide novel capabilities to modify and transform their molecular structure, such as, e.g., radiation-induced polymerization [23].

Atomic clusters have proven to be well suited to investigate the light-induced dynamics in nanosized samples, because their size can be easily varied from a few up to $\sim 10^{7}$ atoms [24,25]. The light-induced disintegration of clusters has been widely used to obtain insight into nonlinear, collective, and correlated many-body processes occurring in nanoplasmas, created through intense light pulses in a wide spectral range from the near-infrared (NIR) to the x-ray domain.

When an atomic cluster is irradiated by an intense NIR laser pulse, electrons occupying valence orbitals in individual atoms are stripped by the strong electric field. However, they remain trapped by the Coulomb potential of the charged cluster, thus forming a transient nanoplasma [26-28]. The trapped delocalized electrons are further heated by the NIR laser via inverse bremsstrahlung (IBS) [29].

In extreme ultraviolet (XUV) and soft-X-ray fields, sequential single-photon ionization of the individual atoms drives the nanoplasma formation, while the IBS contribution is then negligible [30,31]. In contrast, the nanoplasma formation by hard-X-ray pulses is a highly indirect process [21]. At sufficiently high photon energies, the arriving $\mathrm{x}$-ray photons excite electrons predominantly from the atomic inner shells. In light elements the inner-shell electron excitation is followed by Auger decay, resulting in the emission of another quasifree electron. The energetic electrons can excite more electrons through sequential electron impact ionization which proceeds until the impact energies of the electrons are so low that no further ionization is possible. These low-energy electrons are then trapped within the highly charged cluster forming the electronic nanoplasma. Such indirect ionization processes are specific to the hard-x-ray regime. This raises an interesting question: Does the disintegration process of hard-X-ray excited atomic clusters reveal features that differ from the standard disintegration path observed in earlier experiments with soft $\mathrm{x}$ rays? These experiments showed that Coulomb explosion [32] and hydrodynamic expansion [33,34] drive the disintegration of soft-x-ray irradiated clusters $[35,36]$. The electron-ion recombination process, the contribution of which becomes significant on long cluster expansion timescales, plays a crucial role in the efficient charge redistribution in the cluster. As a result, the number of ions with higher charge states is reduced, when compared to the instant just after the ionizing light pulse $[19,37]$. Recent studies in the XUV regime [38-40] reported that a large number of Rydberg atoms are formed during the first few picoseconds of the cluster expansion, due to electron-ion recombination.

For the purpose of achieving a control over the disintegration dynamics within x-ray irradiated nanosized objects, a detailed understanding of all contributing processes and their relevant timescales is very important. Therefore, in the present paper, we study the expansion of argon clusters, following their ionization with a hard-x-ray pulse of moderate fluence, in a time-resolved way. We use a NIR laser as a probe, and record time-resolved electron and ion spectroscopy data. The measurement data are compared with dedicated simulation results. Below we report in detail on the

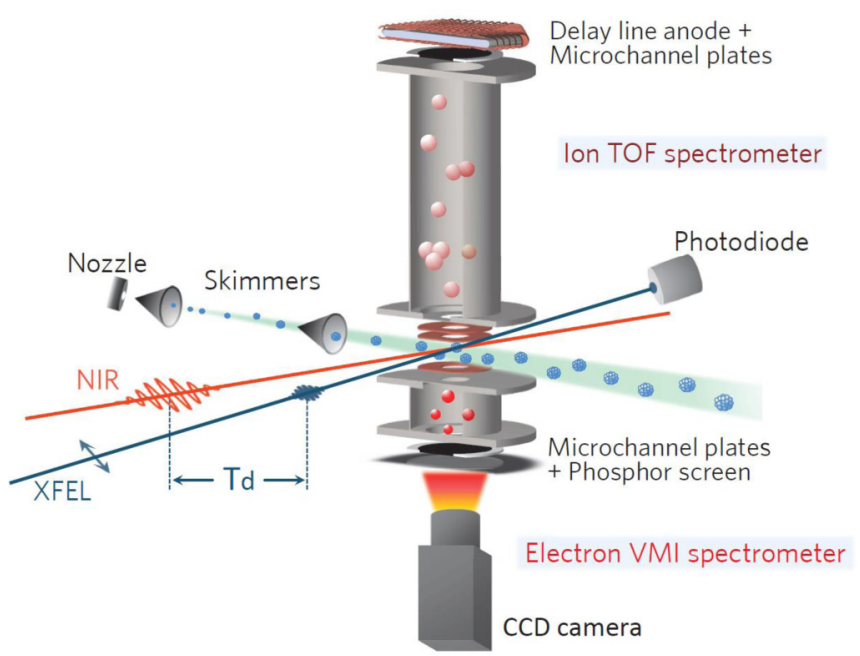

FIG. 1. Experimental scheme for the XFEL-pump-NIR-probe measurement at the SPring-8 Angstrom Compact Free Electron Laser facility. The XFEL and NIR laser were focused into the interaction region, and crossed with the cluster beam from the cluster source through two skimmers. The relative intensity per XFEL pulse was measured by a p-intrinsic-n photodiode located downstream of the interaction point. The produced ions were accelerated by a time-of-flight spectrometer, and detected by a detector consisting of a delay-line anode and two microchannel plates (MCPs). The emitted electrons were recorded by a velocity-map-imaging spectrometer, which consists of two MCPs, a phosphor screen, and a CCD camera.

experimental and simulation scheme, and present the results obtained.

\section{EXPERIMENT}

The experimental setup is schematically shown in Fig. 1. The experiments were performed at the experimental hutch 3 of beamline 3 [41] of the XFEL facility in Japan, the SPring-8 Angstrom Compact free-electron LAser (SACLA) [2]. The x-ray source produced pulses of $5.5-\mathrm{keV}$ photons with $\approx 33-\mathrm{eV}$ [full width at half maximum (FWHM)] photon bandwidth. The repetition rate of the XFEL pulses was $30 \mathrm{~Hz}$. The pulse duration was estimated to be $10 \mathrm{fs}$ (FWHM) [42]. The XFEL beam was focused on the interaction point by Kirkpatrick-Baez mirrors [43]. The focused XFEL beam size was measured to be $\approx 1 \mu \mathrm{m}$ (FWHM). The pulse energies were measured shot-by-shot by the beam-position monitor [44] located upstream of the beamline and calibrated by a calorimeter. The measured value was on average $230 \mu \mathrm{J}$ per pulse. The pulse energy was not measured at the interaction point but upstream. The relative intensity per XFEL pulse was measured by a $\mathrm{p}$-intrinsic-n photodiode located downstream of the interaction point. The shot-to-shot pulse energy fluctuation was $\pm 12 \%$ ( $24 \%$ FWHM). The average peak fluence of the XFEL pulses at the interaction point was determined to be $4.1 \mu \mathrm{J} / \mu \mathrm{m}^{2}$ via an established calibration method using the fragment yields of oligomer ions [22].

The clusters were prepared by an adiabatic expansion of argon gas through a $250-\mu \mathrm{m}$ nozzle at $300 \mathrm{~K}$. The stagnation pressure was $1.1 \mathrm{MPa}$, and the average cluster 
size was estimated to be $\langle N\rangle \sim 1000$ atoms, according to a well-known scaling law [45]. The cluster beam was collimated by two skimmers at 20 and $400 \mathrm{~mm}$ from the nozzle. The inner diameters of the first and second skimmers were 0.5 and $2 \mathrm{~mm}$, respectively. The distance between the second skimmer and the interaction point was $250 \mathrm{~mm}$. The cluster beam at the reaction point was estimated to be $\approx 2 \mathrm{~mm}$ (FWHM), i.e., shorter than the Rayleigh length of $\approx 8 \mathrm{~mm}$. Thus, the ion source volume had a cylindrical shape of $\approx 1 \mu \mathrm{m}$ in diameter and $\approx 2 \mathrm{~mm}$ along the XFEL beam. At the available XFEL fluence, any sequential two-photon ionization of individual atoms in the cluster was negligible. In total, about ten atoms in $\mathrm{Ar}_{1000}$ were photoionized during the exposure to an XFEL pulse. These numbers are several orders of magnitude larger than those achieved with synchrotron radiation experiments [46] and one order less than those in typical XFEL experiments [21].

A NIR laser with a wavelength of $800 \mathrm{~nm}$ was used to probe the expansion of XFEL-ionized clusters. The NIR laser with a focal size of $200 \mu \mathrm{m}$ (FWHM) overlapped with the XFEL beam focus with a small crossing angle (less than $1^{\circ}$ ) at the interaction point. The pulse duration of the NIR laser was $82 \mathrm{fs}$ (FWHM) and the intensity was $5.0 \times 10^{12} \mathrm{~W} / \mathrm{cm}^{2}$. At such low NIR intensity, the ponderomotive energy is two orders of magnitude below the ionization potential of atomic argon [47]. This implies that the NIR pulse cannot ionize ground-state argon atoms. Our experimental data at negative pump-probe delays support this conclusion (not shown).

The intrinsic temporal resolution in such pump-probe experiments, determined by the temporal duration of the XFEL and NIR pulses, is significantly deteriorated by a temporal jitter between the two pulses [48]. In a separate experiment [49], we measured ion yields from xenon clusters, with an average size of $\langle N\rangle \sim 5000$ atoms, irradiated by the XFEL pulse, ionizing short-lived transient excited states of atomic ions by the NIR probe pulse, employing an arrival pulse timing monitor between the two pulses [48]. The absolute time delay was determined within \pm 20 -fs temporal accuracy and temporal jitter between the two pulses was found to be $\approx 800 \mathrm{fs}$ (FWHM). We also found that the time zero between the XFEL and NIR pulses could drift by up to $\approx 1$ ps when the measurements required a long time. In the present experiment, we did not measure the arrival times between the XFEL and NIR laser pulses. The pump-probe time delay was scanned by using an optical delay line and the temporal overlap between the XFEL and NIR pulses was first found using an ultrafast photodiode with a rise time of $30 \mathrm{ps}$ [50]. In order to refine the temporal overlap between the pump and probe pulses and to reach a subpicosecond timing accuracy, we employed a second optimization step with a fast physical signal, i.e., ion yields from xenon clusters with an average size of $\langle N\rangle \sim 5000$ atoms. The yield of doubly charged ions $\left(\mathrm{Xe}^{2+}\right)$ increases as a function of the time delay within $\approx 1$ ps [49]. Assuming the temporal jitter of $\approx 800$ fs (FWHM) and overlapping the present $\mathrm{Xe}^{2+}$ yield curve to the high temporal accuracy one [49] we could refine time zero. As the result, we reached a subpicosecond timing uncertainty.

In the experiment, we measured the kinetic-energy spectrum of electrons and ionic fragments as a function of the time delay between the XFEL-pump and NIR-probe pulses, using the electron velocity-map-imaging (VMI) technique $[21,22,51]$ and ion time-of-flight (TOF) spectrometer $[18,22,51]$. The electron VMI spectrometer employed was specifically designed for use at the XFEL facility, so that high-energy electrons up to $1 \mathrm{keV}$ could be detected. In the present experiment, the VMI spectrometer was adjusted to measure the electrons with low kinetic energies up to $25 \mathrm{eV}$, in order to focus the detection on the peak formed due to thermal electron emission in a nanoplasma. The ion TOF spectrometer accelerated the produced ions, and a position-sensitive detector constructed with microchannel plates (MCPs) and a delay-line anode (DLA) detected the accelerated ions. The signals obtained from the DLA and MCPs were recorded by a digitizer and analyzed by a software discriminator [51].

\section{THEORY}

In order to theoretically describe NIR probing of XFELpumped clusters, we applied an extended version of the XMDYN code $[52,53]$, the simulation tool used in our previous study [22]. In brief, the XMDYN tool tracks the real-space dynamics of the atoms, atomic ions, and emitted electrons, using the classical molecular-dynamics technique. For the previous study [22], we introduced three extensions of the code, adding phenomena occurring within a weakly excited or ionized cluster: van der Waals interaction, oligomer formation, and charge transfer. All details on the code extension can be found in Ref. [22].

In the context of the current paper, it should be emphasized that the code accounts for two important processes in a dense ionized environment, electron secondary ionization and recombination. However, it does not include the spatial propagation effects of the NIR pulse, assuming everywhere the same NIR intensity during the pulse. Also, since in our calculations only classical particles interact with the NIR field (through the NIR electric field), the tunneling ionization process is not taken into account. Therefore, the simulation cannot treat negative delays (NIR-induced ionization of ground-state atoms). More important for the present paper is that the NIR field can ionize even Rydberg atoms, which we describe classically, only through a strong-field over-the-barrier process. As a consequence, within the present framework only sufficiently weakly bound electrons can be dislodged directly by the NIR field.

\section{RESULTS AND DISCUSSIONS}

The ion yields of various oligomers measured and analyzed in our previous study [22] indicated that the ionization dynamics in argon clusters irradiated by a hard-x-ray FEL pulse of moderate fluence is strongly influenced by chemical processes, distributing the increasing net charge within the sample and inducing bonding reorganization. According to our simulations, at a representative $\mathrm{x}$-ray fluence of $3.5 \mu \mathrm{J} / \mu \mathrm{m}^{2}$, on average $\approx 9$ atomic photoionization events occur within an $\mathrm{Ar}_{1000}$ cluster. They are followed by inner-shell electronic relaxation of the atomic ions, releasing on average $\approx 23$ additional electrons within $\approx 20$ fs via Auger processes. The ejected fast electrons leave the system, but they can also 


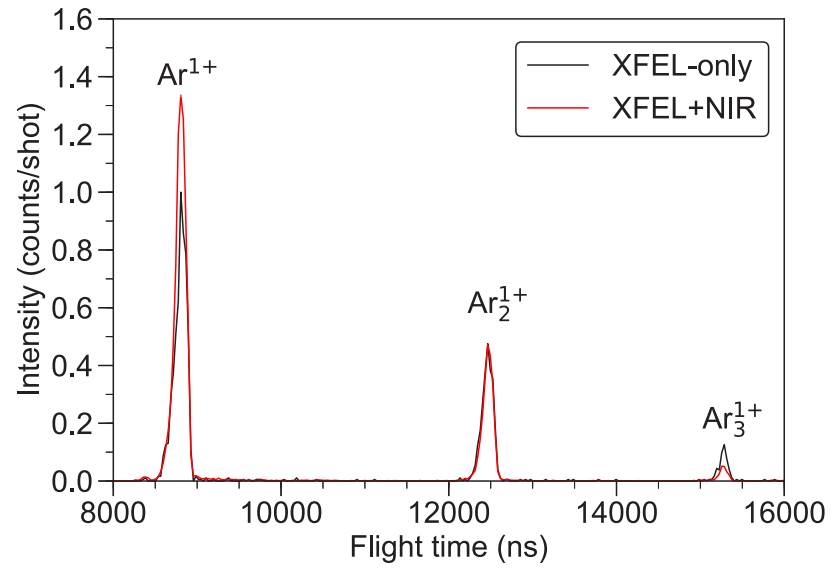

FIG. 2. Time-of-flight spectrum from argon clusters with an average size of $\langle N\rangle \sim 1000$ atoms after irradiation with XFEL pulses $(5.5 \mathrm{keV})$ at an average peak fluence of $4.1 \mu \mathrm{J} / \mu \mathrm{m}^{2}$ (black line). Using an additional weak NIR pulse $(800 \mathrm{~nm})$ with an intensity of $5.0 \times 10^{12} \mathrm{~W} / \mathrm{cm}^{2}$ as a probe pulse, we observe that the $\mathrm{Ar}^{1+}$ ion contribution is enhanced at a time delay of $10 \mathrm{ps}$, whereas the $\mathrm{Ar}_{3}^{1+}$ ion contributions are reduced (red line).

collisionally ionize atoms on their way out of the cluster. On average $\approx 24$ such events happen in $\approx 100$ fs, but approximately only ten of the low-energy electrons also recombine on this timescale. Overall, within $100 \mathrm{fs}$, an $\mathrm{Ar}_{1000}$ cluster reaches a net charge state of about +40 via fast electronic processes, while it contains only $\approx 6$ trapped electrons. Afterwards, the evolution is driven by the fragmentation dynamics of the cluster. While initially the Ar ions are intermixed with the neutral atoms, at $150 \mathrm{fs}$ after the XFEL pulse ions start to leave the system, softening the trapping positive potential. At $400 \mathrm{fs}$, already half of the ionic charges, expanding up to a radius of $30 \AA$, have left the inner part of the cluster (radius of $22 \AA$ ), containing most of the neutral atoms. This finally leads to a gradual decrease of the number of trapped quasifree electrons.

The respective ion TOF spectrum is presented in Fig. 2. It shows the contributions of $\mathrm{Ar}^{1+}, \mathrm{Ar}_{2}^{1+}$, and $\mathrm{Ar}_{3}^{1+}$ ions after irradiation of argon clusters $(\langle N\rangle \sim 1000$ atoms $)$ by XFEL photons of $5.5-\mathrm{keV}$ energy at a peak fluence of $4.1 \mu \mathrm{J} / \mu \mathrm{m}^{2}$ (black line). In the current paper, we investigate the effect of the additional NIR probe pulse on the oligomer distribution. The oligomer peaks were recorded at 10-ps delay (red line). The yield of $\mathrm{Ar}^{1+}$ is clearly enhanced by the action of the probe pulse. This may be attributed to the reionization of excited atoms formed by electron-ion recombination (REAR) during the cluster expansion [39]. In contrast, the $\mathrm{Ar}_{3}^{1+}$ ion yield becomes reduced by the irradiation with the NIR probe pulse. We note that the yields of larger oligomers up to $\mathrm{Ar}_{11}^{1+}$ are also slightly reduced due to the interaction with the probe pulse. The reduction can be ascribed to the fact that the cluster has already fragmented at $10 \mathrm{ps,}$ and the interaction of the weak NIR pulse with dispersed individual oligomer fragments leads to their dissociation [54]. This specific NIR induced channel of fragmentation of the individual oligomer ions also contributes to the enhancement of $\mathrm{Ar}^{1+}$ ions.

The increase of the $\mathrm{Ar}^{1+}$ ion contribution induced by the probe pulse, as shown in Fig. 2, will now be further (a)

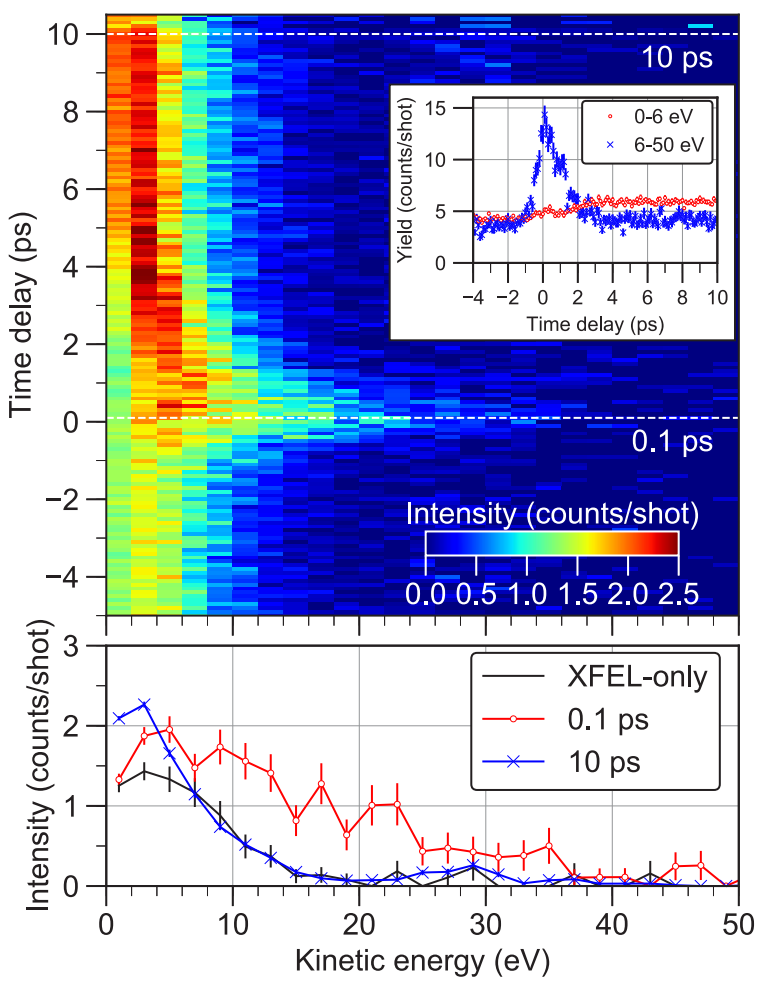

FIG. 3. (a) Kinetic-energy spectra of $\mathrm{Ar}^{1+}$ as a function of the time delay between XFEL pump and NIR probe pulses. The intensity of the NIR pulse is $5.0 \times 10^{12} \mathrm{~W} / \mathrm{cm}^{2}$. (b) Kinetic-energy spectra obtained with the XFEL pulse only and with the additional NIR pulse at time delays of 0.1 and $10 \mathrm{ps}$. The inset of (a) shows the yields of $\mathrm{Ar}^{1+}$ with the kinetic energies of $0-6$ and $6-50 \mathrm{eV}$, respectively, as a function of time delay.

investigated. Figure 3(a) depicts the kinetic-energy spectra of $\mathrm{Ar}^{1+}$ ions measured as a function of the pump-probe time delay. In Fig. 3(b) the kinetic-energy spectra are displayed for the XFEL pump pulse only and for XFEL + NIR pulses at the specific time delays of 0.1 and 10 ps.

At 10-ps time delay we clearly observe the enhancement of the number of $\mathrm{Ar}^{1+}$ ions with low kinetic energy from 0 to $6 \mathrm{eV}$, when compared to the XFEL pump only case. This corresponds to the effect of REAR [39] and the NIR induced oligomer fragmentation [54] discussed above. The inset of Fig. 3(a) shows the yields of $\mathrm{Ar}^{1+}$ ions with kinetic energies of $0-6$ and $6-50 \mathrm{eV}$, respectively, as a function of time delay. In contrast to the gradual increase of the slow $\mathrm{Ar}^{1+}$ ions (with $0-6$-eV kinetic energies) at the times from $\approx 1$ to $\approx 3 \mathrm{ps}$, the signal from fast $\mathrm{Ar}^{1+}$ ions (with 6-50-eV kinetic energies) rapidly increases around $0 \mathrm{ps}$, and then decreases below the signal level from slow ions.

The expansion of an initially overdense nanoplasma enables particularly strong resonant laser energy absorption, once the frequency of the collective electronic dipole mode, the so-called Mie plasmon [27,55], equals the laser frequency. However, the ionization at the present moderate fluence is far too small to create such an overdense nanoplasma inside the $\mathrm{Ar}_{1000}$ clusters. This is also consistent with the observation that the enhancement was not significantly delayed with respect to time zero. 


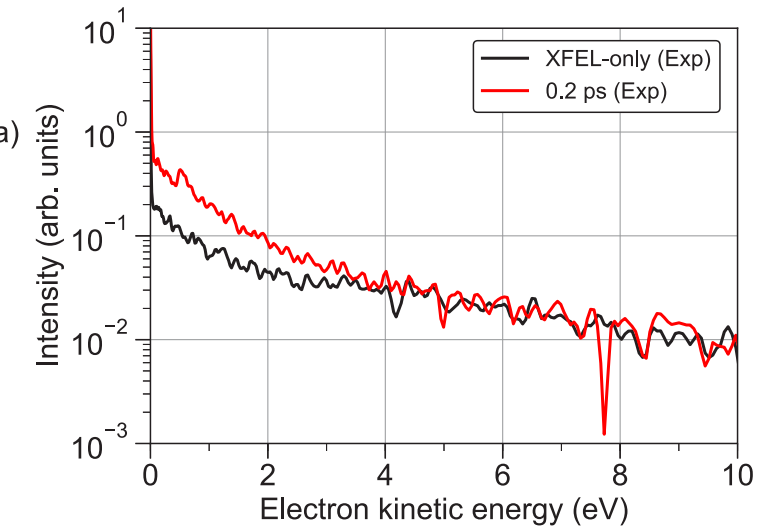

(b)

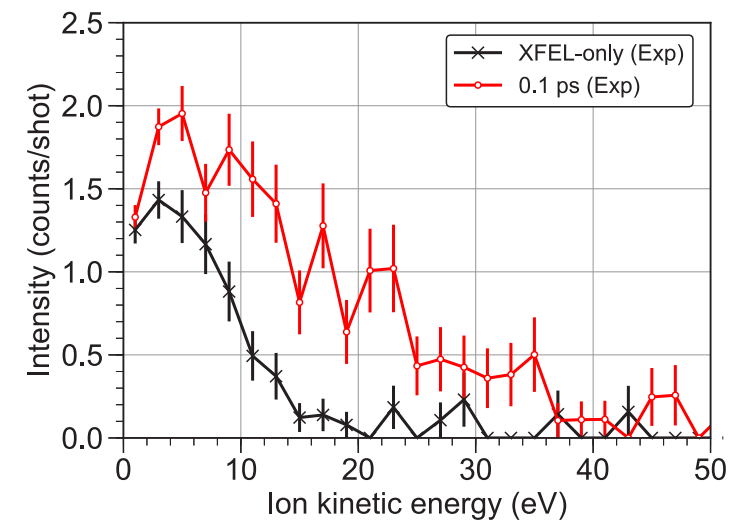

(c)

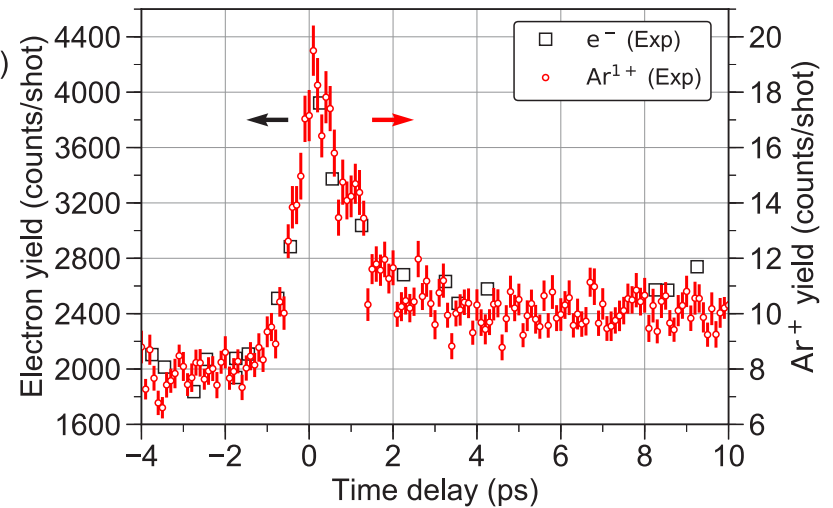

FIG. 4. Electron and ion spectroscopy data obtained at an XFEL peak fluence of $4.1 \mu \mathrm{J} / \mu \mathrm{m}^{2}$ and an NIR intensity of $5.0 \times 10^{12} \mathrm{~W} / \mathrm{cm}^{2}$, with kinetic-energy spectra of (a) electrons and (b) $\mathrm{Ar}^{1+}$ ions and with (c) yields of electron and $\mathrm{Ar}^{1+}$ ion distributions as a function of the time delay between XFEL pump and NIR probe pulses.

On the other hand, similar effects were observed in earlier XUV-pump-NIR-probe experiments [56], namely, the rapid enhancement around 0 ps can be interpreted as resulting from (electron) cascade ionization after the generation of a very few quasifree electrons by the XFEL pulses. Therefore, the rapid decrease of the enhancement does not imply a complete disintegration of the cluster. Instead, it reflects the loss of the small number of quasifree electrons from within the slowly expanding neutral cluster core. This decrease of electron density occurring on a timescale of about 1 ps is caused by the weakening of the trapping cluster potential while the ions move away. (a)

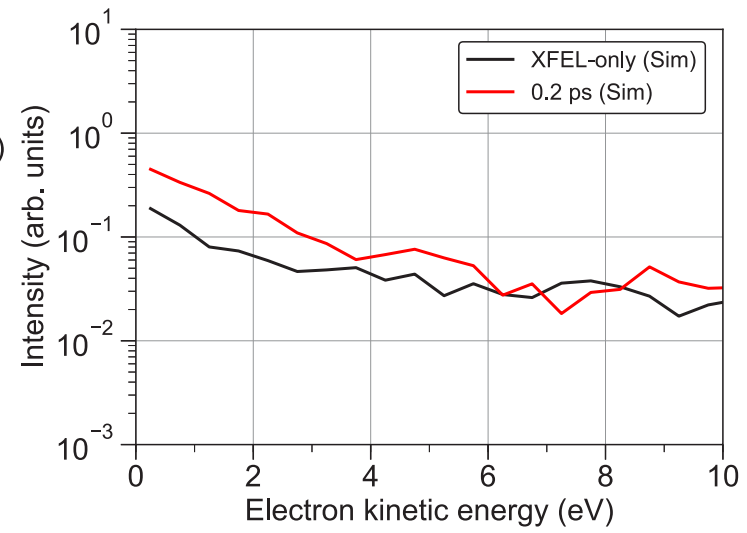

(b)
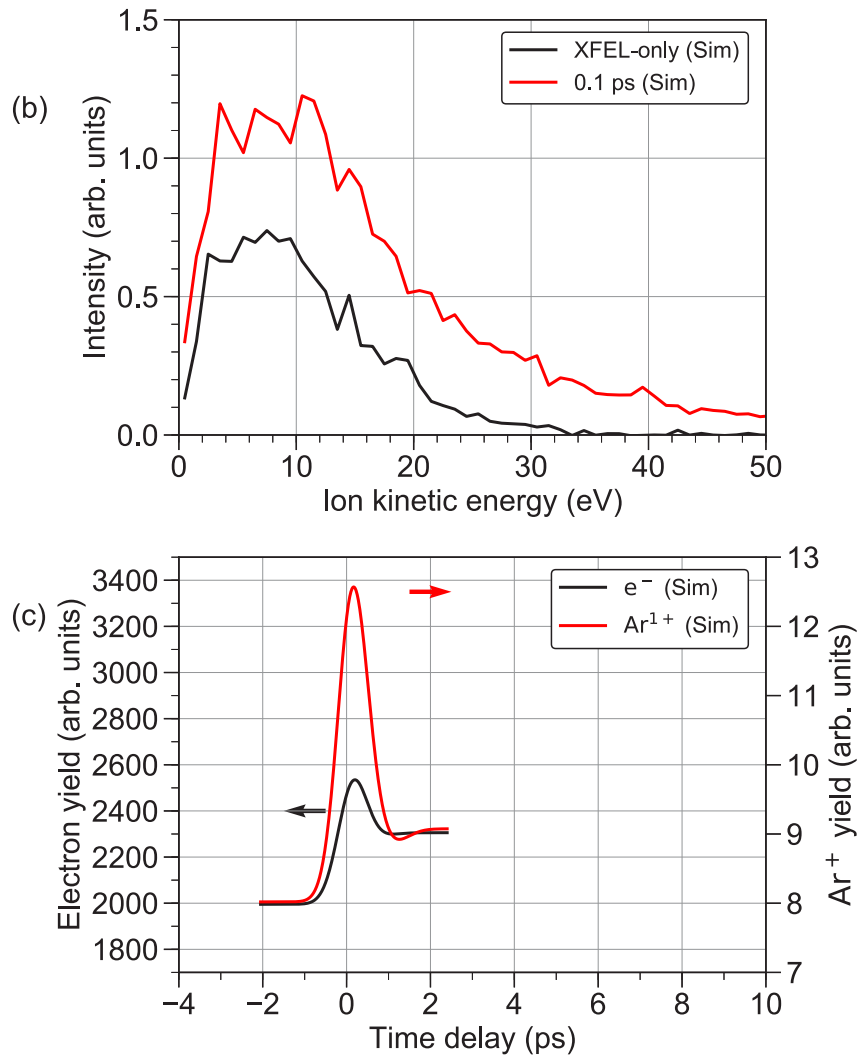

FIG. 5. Theoretical simulations with XMDYN code for electron and ion spectroscopy data obtained at the XFEL peak fluence of $4.1 \mu \mathrm{J} / \mu \mathrm{m}^{2}$ and the NIR intensity of $5.0 \times 10^{12} \mathrm{~W} / \mathrm{cm}^{2}$, with kinetic energy spectra of (a) electrons and (b) $\mathrm{Ar}^{1+}$ ions and with (c) yields of electron and $\mathrm{Ar}^{1+}$ ion distributions as a function of the time delay between XFEL pump and NIR probe pulses. The simulation data take into consideration the focal volume integration.

In order to understand the mechanisms underlying the effects observed, we performed corresponding calculations with XMDYN. The comparison between experimental and theoretical data is presented in Figs. 4 and 5. The experimentally measured kinetic-energy spectrum of electrons for the "XFEL pump only" case was well reproduced by our simulations in previous studies [21,22]. We note that the contributions of LMM Auger electrons (electrons released from the $\mathrm{M}$ shell in an Auger decay when another M-shell electron fills an L-shell vacancy) [21] have been observed at $\approx 200 \mathrm{eV}$ when 
the VMI spectrometer was adjusted to measure the electrons with kinetic energies up to $500 \mathrm{eV}$. Both experimental data and simulations show a thermal electron emission peak at kinetic energies close to zero. The irradiation with an additional weak NIR probe pulse at 0.2-ps time delay leads to an enhancement of the number of thermally emitted electrons [Figs. 4(a) and 5(a)]. This is to be expected as the quasifree electrons in the XFEL-ionized clusters efficiently absorb energy from the electric field of the NIR probe pulse.

In Figs. 4(b) and 5(b) we show $\mathrm{Ar}^{1+}$ kinetic-energy distributions for both the XFEL pump only case and the XFEL-pump-NIR-probe case, at 0.1-ps time delay [see also Fig. 3(b)]. As already discussed in Ref. [22], in the XFEL pump only case, the maximal energy ranges simulated and measured for Ar ions agree with each other; however, the shapes of the curves differ. This discrepancy may be due to the effect of quantum-mechanical valence electron delocalization [57], which can lead to a more efficient redistribution of kinetic energies between ionic fragments [22]. This effect cannot be included in a classical molecular-dynamics model. In case of XFEL + NIR irradiation, this discrepancy disappears, as in the expanding medium individual ionic fragments become increasingly isolated. Coulomb repulsion then drives the separation of the ionic fragments and no valence-electronmediated energy transfer mechanism between ionic fragments is possible. Accordingly, the measured and simulated kineticenergy distributions for $\mathrm{Ar}^{1+}$ are in a good agreement in this case.

Figures 4(c) and 5(c) show the yields of electron and $\mathrm{Ar}^{1+}$ ions as a function of time delay between pump and probe pulses. The rapid enhancements of electron and $\mathrm{Ar}^{1+}$ yields at $\approx 0.1$ ps are well reproduced by our XMDYN simulations. The simulation results indicate that the average number of quasifree electrons inside the inner part of the cluster (containing most of the neutral atoms) decreases below 1 within 1 ps. The higher yields of the finally produced electrons and $\mathrm{Ar}^{1+}$ ions after $\approx 3$ ps observed experimentally may be attributed to the reionization of highly excited (Rydberg) atoms formed during the electron-ion recombination within the expanding cluster [19,37-40].

Also, when comparing the shape of the yield curves, we note that the theory yields decrease more rapidly than the experimental ones after reaching the peak maximum. This discrepancy may be attributed to the effect of the direct ionization by the NIR field. Electrons that have undergone recombination can be reionized, and then further heated, by the NIR field (see Ref. [40]). Overall, this leads to an increased energy absorption rate of the NIR field. However, in our simulations NIR-driven reionization is limited to over-thebarrier ionization, as tunneling ionization is not included.

\section{SUMMARY}

We have presented the results of a time-resolved XFELpump-NIR-probe study of the disintegration processes within argon clusters ionized by hard-X-ray FEL pulses of moderate fluence. The expanding clusters were probed with a NIR laser pulse on timescales from -4 to $10 \mathrm{ps}$. In addition to the observed increasing number of singly charged atomic ions, originating from the ionization of Rydberg atoms formed through electron-ion recombination, we note a decrease of oligomer yields. The latter is due to the interaction of oligomers with the NIR probe pulse, leading to their dissociation. At time delays between -1 and 2 ps, efficient absorption of the NIR laser energy occurs, in spite of the fact that the NIR intensity is too low to trigger optical tuneling ionization of groundstate Ar atoms. Our molecular-dynamics simulations capture the essential features of the energy absorption from the NIR field by the electron nanoplasma within the cluster. During the cluster expansion, two other important processes-direct NIR-induced ionization and electron-ion recombination-are also present and compete with each other, enhancing energy absorption from the NIR laser pulse. In conclusion, probing with weak NIR pulses is a versatile method and can be widely applied to sensitively probe the electron and nuclear dynamics within clusters. The method works also at a low degree of ionization and at low trapped-electron densities, when the overall dynamics depend strongly on chemical processes occurring.

Our observations on the NIR interaction with expanding atomic clusters are similar to observations by other groups, which studied fragmenting clusters excited by soft-x-ray pulses with optical probes. This suggests that the relaxation dynamics of nano-objects after x-ray excitation displays universal features, over a wide photon energy range, and paves the way to achieving control over the disintegration dynamics of nanosized objects induced by x-ray pulses, which may potentially deliver novel capabilities to modify and transform their molecular structure, such as, e.g., radiation-induced polymerization [23].

\section{ACKNOWLEDGMENTS}

We are grateful to the late Makoto Yao for his invaluable contributions to the present paper. The experiments were performed at SACLA with the approval of Japan Synchrotron Radiation Research Institute and the program review committee (No. 2014A8040). This paper was supported by the X-ray Free Electron Laser Utilization Research Project and the X-ray Free Electron Laser Priority Strategy Program of the Ministry of Education, Culture, Sports, Science, and Technology of Japan (MEXT); by the Proposal Program of SACLA Experimental Instruments of RIKEN; by the Japan Society for the Promotion of Science (JSPS) KAKENHI Grants No. JP21244042, No. JP23241033, No. JP15K17487, and No. 16K05016; by MEXT KAKENHI Grant No. JP22740264; by the IMRAM project; and by the National Natural Science Foundation of China Grant No. 11604003. H.F. and K.U. acknowledge "Dynamic Alliance for Open Innovation Bridging Human, Environment and Materials." K.N. and S.W. acknowledge the Research Program "Dynamic Alliance for Open Innovation Bridging Human, Environment and Materials" in "Network Joint Research Center for Materials and Devices." S.M. acknowledges JSPS KAKENHI Grant No. JP11F01028. T.N. acknowledges the Research Program for Next Generation Young Scientists "Dynamic Alliance for Open Innovation Bridging Human, Environment and Materials" in "Network Joint Research Center for Materials and Devices." D.I. and Y.I. acknowledge Institute of Multidisciplinary Research for Advanced Materials project (IMRAM), Tohoku University. 
[1] P. Emma, R. Akre, J. Arthur, R. Bionta, C. Bostedt, J. Bozek, A. Brachmann, P. Bucksbaum, R. Coffee, F.-J. Decker et al., Nat. Photon. 4, 641 (2010).

[2] T. Ishikawa, H. Aoyagi, T. Asaka, Y. Asano, N. Azumi, T. Bizen, H. Ego, K. Fukami, T. Fukui, Y. Furukawa et al., Nat. Photon. 6, 540 (2012).

[3] H. N. Chapman, P. Fromme, A. Barty, T. A. White, R. A. Kirian, A. Aquila, M. S. Hunter, J. Schulz, D. P. DePonte, U. Weierstall et al., Nature (London) 470, 73 (2011).

[4] M. M. Seibert, T. Ekeberg, F. R. N. C. Maia, M. Svenda, J. Andreasson, O. Jonsson, D. Odić, B. Iwan, A. Rocker, D. Westphal et al., Nature (London) 470, 78 (2011).

[5] L. Redecke, K. Nass, D. P. DePonte, T. A. White, D. Rehders, A. Barty, F. Stellato, M. Liang, T. R. M. Barends, S. Boutet et al., Science 339, 227 (2013).

[6] R. Mankowsky, A. Subedi, M. Först, S. O. Mariager, M. Chollet, H. T. Lemke, J. S. Robinson, J. M. Glownia, M. P. Minitti, A. Frano et al., Nature (London) 516, 71 (2014).

[7] K. H. Kim, J. G. Kim, S. Nozawa, T. Sato, K. Y. Oang, T. W. Kim, H. Ki, J. Jo, S. Park, C. Song et al., Nature (London) 518, 385 (2015).

[8] P. Wernet, K. Kunnus, I. Josefsson, I. Rajkovic, W. Quevedo, M. Beye, S. Schreck, S. Grübel, M. Scholz, D. Nordlund et al., Nature (London) 520, 78 (2015).

[9] L. Young, E. P. Kanter, B. Krässig, Y. Li, A. M. March, S. T. Pratt, R. Santra, S. H. Southworth, N. Rohringer, L. F. DiMauro et al., Nature (London) 466, 56 (2010).

[10] S. M. Vinko, O. Ciricosta, B. I. Cho, K. Engelhorn, H.-K. Chung, C. R. D. Brown, T. Burian, J. Chalupský, R. W. Falcone, C. Graves et al., Nature (London) 482, 59 (2012).

[11] H. Yoneda, Y. Inubushi, K. Nagamine, Y. Michine, H. Ohashi, H. Yumoto, K. Yamauchi, H. Mimura, H. Kitamura, T. Katayama et al., Nature (London) 524, 446 (2015).

[12] I. S. Ko, H.-S. Kang, H. Heo, C. Kim, G. Kim, C.-K. Min, H. Yang, S. Y. Baek, H.-J. Choi, G. Mun et al., Appl. Sci. 7, 479 (2017).

[13] T. Tschentscher, C. Bressler, J. Grünert, A. Madsen, A. P. Mancuso, M. Meyer, A. Scherz, H. Sinn, and U. Zastrau, Appl. Sci. 7, 592 (2017).

[14] C. J. Milne, T. Schietinger, M. Aiba, A. Alarcon, J. Alex, A. Anghel, V. Arsov, C. Beard, P. Beaud, S. Bettoni et al., Appl. Sci. 7, 720 (2017)

[15] E. A. Seddon, J. A. Clarke, D. J. Dunning, C. Masciovecchio, C. J. Milne, F. Parmigiani, D. Rugg, J. C. H. Spence, N. R. Thompson, K. Ueda et al., Rep. Prog. Phys. 80, 115901 (2017).

[16] L. Young, K. Ueda, M. Gühr, P. H. Bucksbaum, M. Simon, S. Mukamel, N. Rohringer, K. C. Prince, C. Masciovecchio, M. Meyer et al., J. Phys. B 51, 032003 (2018).

[17] B. Rudek, S.-K. Son, L. Foucar, S. W. Epp, B. Erk, R. Hartmann, M. Adolph, R. Andritschke, A. Aquila, N. Berrah et al., Nat. Photon. 6, 858 (2012).

[18] H. Fukuzawa, S.-K. Son, K. Motomura, S. Mondal, K. Nagaya, S. Wada, X.-J. Liu, R. Feifel, T. Tachibana, Y. Ito et al., Phys. Rev. Lett. 110, 173005 (2013).

[19] H. Thomas, A. Helal, K. Hoffmann, N. Kandadai, J. Keto, J. Andreasson, B. Iwan, M. Seibert, N. Timneanu, J. Hajdu et al., Phys. Rev. Lett. 108, 133401 (2012).

[20] T. Gorkhover, M. Adolph, D. Rupp, S. Schorb, S. W. Epp, B. Erk, L. Foucar, R. Hartmann, N. Kimmel, K.-U. Kühnel et al., Phys. Rev. Lett. 108, 245005 (2012).
[21] T. Tachibana, Z. Jurek, H. Fukuzawa, K. Motomura, K. Nagaya, S. Wada, P. Johnsson, M. Siano, S. Mondal, Y. Ito et al., Sci. Rep. 5, 10977 (2015).

[22] Y. Kumagai, Z. Jurek, W. Xu, H. Fukuzawa, K. Motomura, D. Iablonskyi, K. Nagaya, S. Wada, S. Mondal, T. Tachibana et al., Phys. Rev. Lett. 120, 223201 (2018).

[23] Radiation Curing, edited by S. P. Pappas (Springer, New York, 1992).

[24] U. Saalmann, C. Siedschlag, and J. M. Rost, J. Phys. B 39, R39 (2006).

[25] T. Fennel, K.-H. Meiwes-Broer, J. Tiggesbäumker, P.-G. Reinhard, P. M. Dinh, and E. Suraud, Rev. Mod. Phys. 82, 1793 (2010).

[26] E. M. Snyder, S. A. Buzza, and A. W. Castleman, Jr., Phys. Rev. Lett. 77, 3347 (1996).

[27] T. Ditmire, T. Donnelly, A. M. Rubenchik, R. W. Falcone, and M. D. Perry, Phys. Rev. A 53, 3379 (1996).

[28] Y. L. Shao, T. Ditmire, J. W. G. Tisch, E. Springate, J. P. Marangos, and M. H. R. Hutchinson, Phys. Rev. Lett. 77, 3343 (1996).

[29] V. Krainov and M. Smirnov, Phys. Rep. 370, 237 (2002).

[30] C. Bostedt, H. Thomas, M. Hoener, E. Eremina, T. Fennel, K.-H. Meiwes-Broer, H. Wabnitz, M. Kuhlmann, E. Plönjes, K. Tiedtke et al., Phys. Rev. Lett. 100, 133401 (2008)

[31] M. Arbeiter and T. Fennel, Phys. Rev. A 82, 013201 (2010).

[32] I. Last and J. Jortner, Phys. Rev. A 62, 013201 (2000).

[33] M. Rusek and A. Orłowski, Phys. Rev. A 71, 043202 (2005).

[34] B. Ziaja, H. Wabnitz, E. Weckert, and T. Möller, New J. Phys. 10, 043003 (2008).

[35] H. Thomas, C. Bostedt, M. Hoener, E. Eremina, H. Wabnitz, T. Laarmann, E. Plönjes, R. Treusch, A. R. B. de Castro, and T. Möller, J. Phys. B 42, 134018 (2009).

[36] M. Arbeiter and T. Fennel, New J. Phys. 13, 053022 (2011).

[37] M. Hoener, C. Bostedt, H. Thomas, L. Landt, E. Eremina, H. Wabnitz, T. Laarmann, R. Treusch, A. R. B. de Castro, and T. Möller, J. Phys. B 41, 181001 (2008).

[38] B. Schütte, M. Arbeiter, T. Fennel, M. J. J. Vrakking, and A. Rouzée, Phys. Rev. Lett. 112, 073003 (2014).

[39] B. Schütte, F. Campi, M. Arbeiter, T. Fennel, M. J. J. Vrakking, and A. Rouzée, Phys. Rev. Lett. 112, 253401 (2014).

[40] B. Schütte, T. Oelze, M. Krikunova, M. Arbeiter, T. Fennel, M. J. J. Vrakking, and Arnaud Rouzée, New J. Phys. 17, 033043 (2015).

[41] M. Yabashi, H. Tanaka, T. Tanaka, H. Tomizawa, T. Togashi, M. Nagasono, T. Ishikawa, J. R. Harries, Y. Hikosaka, A. Hishikawa et al., J. Phys. B 46, 164001 (2013).

[42] Y. Inubushi, K. Tono, T. Togashi, T. Sato, T. Hatsui, T. Kameshima, K. Togawa, T. Hara, T. Tanaka, H. Tanaka et al., Phys. Rev. Lett. 109, 144801 (2012).

[43] H. Yumoto, H. Mimura, T. Koyama, S. Matsuyama, K. Tono, T. Togashi, Y. Inubushi, T. Sato, T. Tanaka, T. Kimura et al., Nat. Photon. 7, 43 (2013).

[44] K. Tono, T. Kudo, M. Yabashi, T. Tachibana, Y. Feng, D. Fritz, J. Hastings, and T. Ishikawa, Rev. Sci. Instrum. 82, 023108 (2011).

[45] U. Buckand R. Krohne, J. Chem. Phys. 105, 5408 (1996).

[46] H. Murakami, K. Nagaya, Y. Ohmasa, H. Iwayama, and M. Yao, J. Chem. Phys. 126, 054306 (2007). 
[47] S. F. J. Larochelle, A. Talebpour, and S. L. Chin, J. Phys. B 31, 1215 (1998).

[48] T. Katayama, S. Owada, T. Togashi, K. Ogawa, P. Karvinen, I. Vartiainen, A. Eronen, C. David, T. Sato, K. Nakajima et al., Structural Dynamics 3, 034301 (2016).

[49] Y. Kumagai, H. Fukuzawa, K. Motomura, D. Iablonskyi, K. Nagaya, S. Wada, Y. Ito, T. Takanashi, Y. Sakakibara, D. You et al., Phys. Rev. X 8, 031034 (2018).

[50] L.-P. Oloff, A. Chainani, M. Matsunami, K. Takahashi, T. Togashi, H. Osawa, K. Hanff, A. Quer, R. Matsushita, R. Shiraishi et al., Sci. Rep. 6, 35087 (2016).

[51] H. Fukuzawa, K. Nagaya, and K. Ueda, Nucl. Instrum. Methods Phys. Res., Sect. A 907, 116 (2018).
[52] B. F. Murphy, T. Osipov, Z. Jurek, L. Fang, S.-K. Son, M. Mucke, J. H. D. Eland, V. Zhaunerchyk, R. Feifel, L. Avaldi et al., Nat. Commun. 5, 4281 (2014).

[53] Z. Jurek, S.-K. Son, B. Ziaja, and R. Santra, J. Appl. Crystallogr. 49, 1048 (2016).

[54] B. Schütte, T. Oelze, M. Krikunova, M. Arbeiter, T. Fennel, M. J. J. Vrakking, and A. Rouzée, J. Phys. B 48, 185101 (2015).

[55] G. Mie, Ann. Phys. (Leipzig) 330, 377 (1908).

[56] B. Schütte, M. Arbeiter, A. Mermillod-Blondin, M. J. J. Vrakking, A. Rouzée, and T. Fennel, Phys. Rev. Lett. 116, 033001 (2016).

[57] D. Hrivňák and R. Kalus, Chem. Phys. 264, 319 (2001). 